\title{
Enseignement de la culture entrepreneuriale et influence de la méthode pédagogique
}

\author{
Saloua BANI \\ Enseignante chercheur ISET Nabeul ISET de Nabeul - Campus Universitaire-8000 Nabeul-
}

\begin{abstract}
This research focuses on the influence of the teaching method used to teach entrepreneurial culture in ISET. It focuses specifically on the achievements of students following a course of entrepreneurial culture. The harvest data was made by a addressed to students of third year management science during the month of May 2013 questionnaire.

The results showed that other knowledge, this course leads to effects on behavioral development of students.
\end{abstract}

\section{Introduction}

Depuis quelques années les Instituts Supérieures des Etudes Technologiques (I.S.E.T), comme toutes les universités Tunisiennes ont suivi le régime Licence-Master-Doctorat (L.M.D). Le régime LMD pour les ISET a été décrit par des plans d'études des différentes licences approuvés par la Commission Nationale Sectorielle des Etudes Technologiques. Ces plans d'études ont intégré l'unité d'enseignement culture entrepreneuriale pour toutes les spécialités confondues.

Cette étude s'intéresse à l'influence de la méthode et du processus d'enseignement sur les acquis comportementaux des étudiants en matière de culture entrepreneuriale. Cette recherche porte spécifiquement sur l'analyse d'un cours de culture entrepreneuriale offerts à des étudiants appartenant au département sciences de gestion.

Dans un premier temps, les objectifs et la structure du cours seront exposés. Puis, il sera question, brièvement de la genèse de l'entrepreneurship pour ensuite aborder la formule et de la méthode pédagogique entrepreneuriale employées dans ce cours, lesquelles constituent le cadre de référence théorique qui permet d'expliquer le processus d'apprentissage et les performances obtenues par les participants. Les différentes analyses statistiques employées dans cette recherche et les résultats montrent que cette méthode favorise les apprentissages et que les facteurs d'influençant ces apprentissages sont le mini projet, plus spécifiquement les nombreuses rencontres avec des intervenants du milieu des affaires et l'encadrement des enseignants.

\section{Le Contexte Economique Et L'esprit D'entreprendre}

Les entreprises tunisiennes sont de plus en plus dans un contexte d"'incertitude et de compétitivité, elles ont davantage besoin d'innover pour survivre. En effet, les changements qui surviennent dans l'environnement font pression sur celle-ci et incitent les gestionnaires à développer une attitude et des comportements entrepreneuriaux et des solutions créatives aux différents problèmes qu'ils engendrent (Gibb, 2001).

Dans une nouvelle approche adoptée l'entrepreneurship ne se limite plus au cadre traditionnel de la création d'entreprises, mais porte également sur la création d'une culture entrepreneuriale et le développement d'habiletés entrepreneuriales chez les individus au sein des organisations ou à l'extérieur de celles-ci (Gibb, 2001; Jack \& Anderson, 1999). Une distinction nette peut donc être faite entre l'esprit d'entreprise et l'esprit d'entreprendre.

"...l'esprit d'entreprise semble renvoyer à un ensemble d'attitudes générales positives vis-à-vis de la notion d'entreprise et de celle d'entrepreneur alors que l'esprit d'entreprendre est davantage relié à la prise d'initiative et à l'action (Léger-Jarniou, $2001: 5$ ).

Le rôle classique des universités est la production et le transfert de la connaissance, se transforme vers un rôle d'entrepreneur selon lequel la formation dispensée aux étudiants ne doit pas demeurer strictement liée à la connaissance, mais doit également conduire à l'expérience.

Dans cette optique, le développement d'attitudes et de comportements entrepreneuriaux et l'application de ceux-ci par les étudiants universitaires deviennent cruciaux puisqu'ils répondent directement aux besoins suscités non seulement par les entreprises, mais de manière beaucoup plus large par la mondialisation (Gibb, 2001).

L'enseignement technologique en Tunisie offre aux étudiants le cours de culture entrepreneuriale dans lequel une approche pédagogique entrepreneuriale est utilisée. Dans la présente recherche nous présentons l'influence de l'approche pédagogique pour l'apprentissage des étudiants de la culture entrepreneuriale. 


\section{Les Objectifs Et La Structure Du Cours}

Ce cours vise à consolider chez les étudiants les habiletés et les connaissances en ce qui a trait, entre autres, à l'entrepreneurship, au travail d'équipe, à l'innovation et à la gestion des connaissances. Les étudiants inscrits à ce cours ont suivi de nombreux autres cours qui leur ont permis d'acquérir des connaissances dans nombre de domaines relatifs à la gestion des affaires. Cependant, seulement quelques uns de ces cours leur ont permis d'appliquer concrètement et en situation réelle les connaissances apprises et ainsi assurer leur intégration.

Les étudiants sont regroupés aléatoirement en équipes de deux à trois individus, sont appelés à préparer un projet réalisable sur le marché tunisien. L'évaluation du projet est faite par les autres équipes.

En plus de ce travail en équipe les étudiants sont invités à des travaux individuels et d'autres optionnels.

La méthode pédagogique entrepreneuriale mise en application dans le cadre à l'étude se rapporte directement au développement de l'esprit d'entreprendre. En d'autres termes, cette méthode s'appuie sur l'enseignement de l'attitude et des comportements propres à un entrepreneur mais aussi à des individus qui ne cherchent pas nécessairement à devenir eux aussi des entrepreneurs.

\section{La Culture Entrepreneuriale}

L'évolution de l'éducation entrepreneuriale s'est réalisée en trois étapes définies par trois approches distinctes. Elle a d'abord été caractérisée comme partie intégrante de l'éducation managériale (Filion,1991). Puis, au fur et à mesure que s'est développé ce champ particulier qu'est l'éducation entrepreneuriale, de nombreux auteurs ont affirmé leur désaccord avec la première approche en soutenant que l'éducation managériale, laquelle se rapporte surtout aux grandes entreprises, diffère largement de l'éducation entrepreneuriale qui focalise bien davantage sur les PME (Filion, 1991). La troisième approche tend à réconcilier les deux premières avec l'intérêt renouvelé que constitue le rôle du leadership dans le changement organisationnel et le changement des structures organisationnelles (Leitch et Harrison, 1999 ;Filion, 1991).

Parallèlement au débat entourant l'éducation managériale et entrepreneuriale, la question des processus d'apprentissage des individus et des organisations a largement influencé la littérature portant sur l'entrepreneurship (Gibb, 1997). C'est précisément en liant avec cet élément qu'une nouvelle approche de l'éducation entrepreneuriale, visant le développement d'attitudes, d'habiletés et de comportements entrepreneuriaux chez les individus à l'intérieur et à l'extérieur des organisations, a été adoptée (Gibb, 2001; Jack \& Anderson, 1999).

« Peut-on enseigner l'entrepreneurship? », une réponse positive peut être donnée, mais à certaines conditions propres au développement de l'esprit entrepreneurial. En effet, l'apprentissage d'une attitude et de comportements entrepreneuriaux, de l'ouverture au changement et de la création de nouvelles idées nécessite un contexte pédagogique particulier, favorable à l'expérimentation par les étudiants (Neunreuther, 1979 cité dans Léger-Jarniou,2001; Gibb, 1992). "L'un des principaux défis de l'enseignement entrepreneurial consiste à intégrer les besoins de l'étudiant à des stratégies d'enseignement efficaces afin d'accroître chez celui-ci sa capacité de s'adapter aux réalités de l'entrepreneurship » (Atlantic Canada Opportunities Agency - Agence de Promotion Économique du Canada Atlantique, (ACOA), $2000: 4$ ).

\section{Methode D'enseignement}

L'enseignement de l'entrepreneurship constitue un élément primordial au développement de la culture et des comportements entrepreneuriaux chez les étudiants. Sur ce point, plusieurs auteurs s'accordent pour reconnaître qu'il n'y a pas de contenu idéal et que les connaissances, habiletés et attitudes varient en fonction des besoins d'apprentissage de l'étudiant. Pour certains, ces besoins ne peuvent s'incarner que dans un contexte d'entreprise et non dans un contexte d'école (Schied-Bienfait, 1999).

Certains autres défendent cependant une autre conception selon laquelle l'apprentissage de l'entrepreneurship est possible et même profitable lorsque réalisé à l'intérieur du cadre universitaire selon une méthode dite entrepreneuriale. L'enseignement de l'entrepreneurship dans les universités est davantage une affaire de méthode plutôt que de contenu ;

La méthode d'enseignement selon le modèle entrepreneurial vise à :

- Apprendre les uns des autres

- Apprendre en faisant

- Apprendre en résolvant des problèmes

- Apprendre des échanges interpersonnels et des débats

- Apprendre en découvrant, l'enseignant étant un guide

- Apprendre des réactions des autres

- Apprendre dans un environnement flexible et informel 
- Apprendre sous la pression des objectifs immédiats

- Apprendre en empruntant aux autres

- Apprendre de ses erreurs

(Gibb, 1992 cité dans Duchéneaut, 1997 : 6)

Pour tester l'influence de cette méthode d'enseignement à développer le comportement entrepreneuriale chez les étudiants, une enquête a été faite sur 90 étudiants de troisième année licence sciences de gestion.

\section{Instrument De Recherche Et Analyse Des Resultats}

La cueillette de données à été réalisée par un questionnaire, ceci il a été représenté en trois axes les suivants :

- L'appréciation globale du cours et les motivations des étudiants,

- Les apprentissages réalisés,

- Les appréciations du cours.

Les questions se résument comme suit :

- Ce cours m'a permis de développer mon réseau de contacts.

- Ce cours m'a permis d'apprendre à travailler en complémentarité dans une

- équipe.

- Ce cours favorise la prise d'initiatives.

- Ce cours permet de développer l'autonomie et/ou la responsabilisation et/ou la proactivité

- Ce cours m'a permis de développer des outils de gestion portant sur le développement de grilles d'analyse.

- Ce cours m'a permis de développer des outils de gestion portant sur la recherche et/ou l'innovation et/ou la gestion des connaissances.

- Les activités réalisées dans le cadre du cours étaient pertinentes.

- Ce cours m’a permis de développer mon assurance.

- J'ai acquis et mis en pratique une méthode de travail.

- J'ai acquis et mis en pratique des outils et des techniques de gestion.

- Ce cours m'a permis de développer mon sens des responsabilités

- Ce cours m'a permis de développer mon initiative.

Les variables seront par la suite présentées en trois familles des variables :

- Variables de perception

- Variables de motivation

- Variables d'attitude

- Variables de contrôle

Tableau 1 : Blocs de variables issus de l'analyse de corrélations

\begin{tabular}{|c|l|}
\hline Blocs des variables & Variables \\
\hline Variables de contrôle & Âge \\
& Sexe \\
& Nationalité \\
\hline Variables de perception & - Ce cours m'a permis de développer mon réseau de contacts. \\
& - Ce cours m'a permis d'apprendre à travailler en complémentarité dans une \\
& - équipe. \\
& - Ce cours favorise la prise d'initiatives. \\
& - Ce cours permet de développer l'autonomie et/ou la responsabilisation et/ou la \\
& proactivité \\
\hline Variables de motivation & - Ce cours favorise la prise d'initiatives. \\
& - Ce cours permet de développer l'autonomie et/ou la responsabilisation et/ou la \\
& proactivité \\
& - Ce cours m'a permis de développer des outils de gestion portant sur le \\
& développement de grilles d'analyse. \\
& - Ce cours m'a permis de développer des outils de gestion portant sur la recherche \\
& et/ou l'innovation et/ou la gestion des connaissances. \\
& - Les activités réalisées dans le cadre du cours étaient pertinentes. \\
& - Ce cours m'a permis de développer mon assurance. \\
\hline Variables d'attitude & - J'ai acquis et mis en pratique une méthode de travail. \\
& - J'ai acquis et mis en pratique des outils et des techniques de gestion. \\
& - Ce cours m'a permis de développer mon sens des responsabilités \\
& - Ce cours m'a permis de développer mon initiative. \\
\hline
\end{tabular}


Une analyse de corrélation s'est avérée intéressante. Puisque la méthode pédagogique entrepreneuriale se base sur un triple apprentissage cognitif - conatif - affectif (Gibb 2001, 1998, 1992; Duchébeaut, 1997; Robinson, Hunt \& Huefner, 1991), il sera pertinent de s'interroger sur la relation entre ces apprentissages et les familles des variables déjà présentées.

En conclusion à travers cette recherche nous avons voulu montré que l'enseignement de la culture entrepreneuriale par la l'approche entrepreneuriale en enseignement à permis aux étudiants de développer non seulement des connaissances mais aussi des comportements d'entrepreneurship. En effet avec les activités réalisées, particulièrement les activités d'équipe, ont fourni aux étudiants un cadre favorable aux apprentissages et au travail. Cependant, il importe de ne pas négliger les autres apprentissages individuels réalisés qui ont permis aux équipes, et aux étudiants, d'accomplir toutes les tâches du mini projet.

L'étude a montré que l'enseignement de ce cours renforce les connaissances, développe l'apprentissage d'ordre personnel, et un appui du savoir faire et du savoir être.

\section{REFERENCES}

[1]. BLOCK, Z. et STUMPF, S.A., 1992. «Entrepreneurship Education Research : Experience and challenge ». In The State of the Art of Entrepreneurship, SEXTON, D.L. et KASARDA, J.D., Boston, PWS- Kent, p. 17-42.

[2]. BROWN, C. 2000. «Entrepreneurial Education Teaching Guide ». Kauffman Center for Entrepreneurial Leadership Clearinghouse on Entrepreneurship Education, décembre 2000, Digest no. 00-7.

[3]. DUCHÉNEAUT, B. 1997. "Entrepreneurship and Higher Education : From Real-Life Context to Pedagogical Challenge ». In Conference Internationalizing Entrepreneurship Education and Training (INTENT). 7e conférence annuelle, Monterey Bay, Californie, Mai 1997, 21 p.

[4]. HILLS G.E. 1998. « Variations On University Entrepreneurship Education : An Empirical Study Of An Evolving Field ». Journal of Business Venturing, vol. 3, p.109-122.

[5]. KLANDT, H. 1993. " Methods of Teaching : What Is Useful For Entrepreneurship Education? ». In Internationalizing Entrepreneurship Education And Training (INTENT), 3e conférence annuelle,Vienne, Autriche, 5-7 juillet, 1993, $10 \mathrm{p}$.

[6]. LEITCH, C. et HARRISON, R. 1999. « A process model for entrepreneurship education and developpement». International Journal of Entrepreneurial Behaviour \& Research, Bradford, vol. 5, no. 3, p. 83-109.

[7]. VERSTRAETE, T. 2001. «Entrepreneuriat: modélisation du phénomène ». Revue de l'entrepreneuriat, vol. 1, no.1, p. 5-24. 